\title{
REABILITAÇÃO ESTÉTICA COM RESINA COMPOSTA EM PACIENTE JOVEM: RELATO DE CASO CLÍNICO
}

\author{
AESTHETIC REHABILITATION WITH COMPOSITE RESIN IN A YOUNG PATIENT: A CASE REPORT
}

\author{
Mayele Rodrigues PEREIRA; Layany Lima BALEEIRO'; Uriel Paulo COELHO²; Natália Galvão GARCIA \\ 1 - Graduanda em Odontologia pela Faculdade Morgana Potrich. \\ 2 - Especializando e Mestrando em Prótese Dentária pela São Leopoldo Mandic, Campinas, São Paulo. \\ 3 - Mestre, Doutora e Pós-doutora em Ciências Odontológicas Aplicadas pela Faculdade de Odontologia de Bauru (FOB-USP). Docente do Curso de \\ Odontologia do Centro Universitário de Lavras (UNILAVRAS-MG).
}

\section{RESUMO}

Objetivo: o presente trabalho tem como objetivo relatar um caso clínico de reabilitação de dentes anteriores com facetas em resina composta. Caso clínico: Paciente do sexo masculino, 23 anos, queixava-se de insatisfação com a estética do seu sorriso. No exame clínico foram observadas restaurações insatisfatórias nas incisais e interproximais dos incisivos centrais superiores. Os elementos 12, 22 e 23 apresentavam-se em desarmonia com a face. Após plano de tratamento, foi realizado registro de cor inicial e três sessões de clareamento dental. Na consulta seguinte foi feita profilaxia com pedra pomes extrafina, confecção da guia de silicone através do enceramento, remoção das resinas antigas, seguido de condicionamento ácido e sistema adesivo. Com a guia de silicone e tiras de poliéster em posição foram realizados incrementos de resina composta de dentina e esmalte. Na sequência os excessos foram removidos com pontas diamantadas multilaminadas finas e extrafinas, verificando os contatos oclusais. Posteriormente, foi realizado polimento com borracha de silicone, discos sof-lex e de feltro, associados à pasta diamantada. Ao final do tratamento obteve-se um sorriso esteticamente harmônico e consequentemente a satisfação por parte do paciente. Conclusão: A reabilitação de dentes anteriores com facetas dentárias em resina composta é um tratamento viável para se alcançar um sorriso harmônico.

PALAVRAS-CHAVE: Estética dentária; Facetas dentárias; Sorriso.

\section{INTRODUÇÃO}

A estética é influenciada por fatores psicossociais, culturais e econômicos, levando as pessoas a buscarem cada vez mais uma alternativa de tratamento para modificar a aparência do seu sorriso ${ }^{1}$.

Alterações como, forma, cor, presença de diastema, perda da estrutura dentária por cárie ou traumatismo, podem provocar uma total desarmonia entre o sorriso e a face ${ }^{2}$.

Essa incessante busca tem resultado em avanços nas propriedades físicas e ópticas dos materiais odontológicos, no desenvolvimento de técnicas mais conservadoras, e consequentemente, na obtenção de resultados de excelência ${ }^{1,2}$.

As resinas compostas apresentam como vantagens, preservação da estrutura dental sadia, capacidade de reprodução do aspecto natural através da técnica de estratificação de cor, possibilidade de reparo, longevidade, entre outras ${ }^{2-4}$. Sendo assim, a técnica restauradora utilizando resinas compostas quando indicadas corretamente é uma alternativa de tratamento de baixo custo bastante satisfatória ${ }^{3-6}$.

Diante desse contexto, este trabalho tem como objetivo relatar um caso clínico de reabilitação estética por meio de resina composta em um paciente com restauração anterossuperior insatisfatória e formas anatômicas inadequadas com intuito de ressaltar que a reabilitação de dentes anteriores com facetas dentárias em resina composta pode ser considerada um tratamento viável para se alcançar um sorriso harmônico.

\section{RELATO DE CASO CLÍNICO}

Paciente do sexo masculino, 23 anos de idade, compareceu à Policlínica do Centro de Ensino Superior Morgana Potrich, Mineiros, GO, Brasil, devido à insatisfação com a estética do seu sorriso (Figura 1A). No exame clínico foi observada a presença de restaurações insatisfatórias quanto à cor e forma nas incisais e interproximais dos incisivos centrais superiores (Figura 1B). Foram realizadas radiografias periapicais e teste de vitalidade, os quais excluíram envolvimento pulpar dos dentes avaliados.

Inicialmente, foi realizada moldagem com alginato e confecção do modelo de gesso para realização do planejamento e enceramento de diagnóstico. Para diagnóstico e estudo das guias de desoclusão durante o enceramento, os modelos de gesso foram montados em articulador semi ajustável (Bioart) com o auxílio do arco facial. Após estabelecido o plano de tratamento, foi realizado clareamento dental com peróxido de hidrogênio à 35\% (Whiteness HP MAXX, FGM, Joinville, SC, Brasil) de acordo com as instruções do fabricante, em três sessões com intervalo semanal entre elas (Figura 2A e B).

Aguardou-se 21 dias para estabilização da cor e com auxílio de uma escala (VITAPAN, Classical, SP, Brasil), sob luz natural, e também pela técnica de incremento de resina na face vestibular, foi realizada a seleção da cor dos dentes (Figura 3A e B). 
Em seguida, o modelo de gesso já com o enceramento foi moldado com silicona pesada e ativador (Optosil ${ }^{\circledR}$ Comfort Putty, Hanau, Alemanha) para obtenção da guia palatina (Figura 4A). A guia de silicona foi levada em posição na cavidade oral, para verificar correta adaptação e remoção dos excessos com lâmina de bisturi 12 C (Solidor, Osasco, São Paulo, SP, Brasil) e retirada em seguida.

Foi feito isolamento absoluto modificado na região anterior com lençol de borracha (Madeitex, São José dos Campos, SP, Brasil) e grampos 14A (Golgran, São Caitano do Sul, SP, Brasil) nos molares superiores (Figura 4B).

As restaurações antigas foram removidas com brocas diamantadas esféricas no 1014 (KG, sorensen, Kotia, Brasil) não sendo realizado nenhum preparo ou desgaste na estrutura dental sadia. Seguido de condicionamento ácido por 30 segundos em esmalte com ácido fosfórico à 37\% (Condac 37\%, FGM, Joinville, SC, Brasil) (Figura 5A) e lavagem por 60 segundos, sempre protegendo os dentes vizinhos (Figura 5B). O sistema adesivo (Adper Single Bond 2 - 3M, São José do Rio Preto, SP Brasil) foi aplicado e fotoativado (fotopolimerizador Emitter C Wireless - Schuster) por 20 segundos (Figura $5 \mathrm{C}$ e D).

A guia de silicona foi levada em posição com incrementos de resinas compostas de esmalte (Resina Filtek Z350 XT, 3M ESPE, São José do Rio Preto, SP, Brasil) e fotoativados por 20 segundos, reproduzindo a face palatina (Figura $6 \mathrm{~A} \mathrm{e} \mathrm{B)}$ ).

Em seguida os incrementos de resina de dentina e corpo (Resina Filtek Z350 XT, 3M ESPE, São José do Rio Preto, SP, Brasil) foram sendo colocados respeitando a técnica de estratificação de cor e reproduzindo a forma dental conforme o enceramento de diagnóstico (Figura 6C). Sob a última camada foi colocado gel hidrossolúvel KY (Semina Indústria e Comércio Ltda, São Paulo, $\mathrm{SP}$, Brasil) para bloqueio do oxigênio, permitindo a fotopolimerização completa da resina composta (Figura 6D).

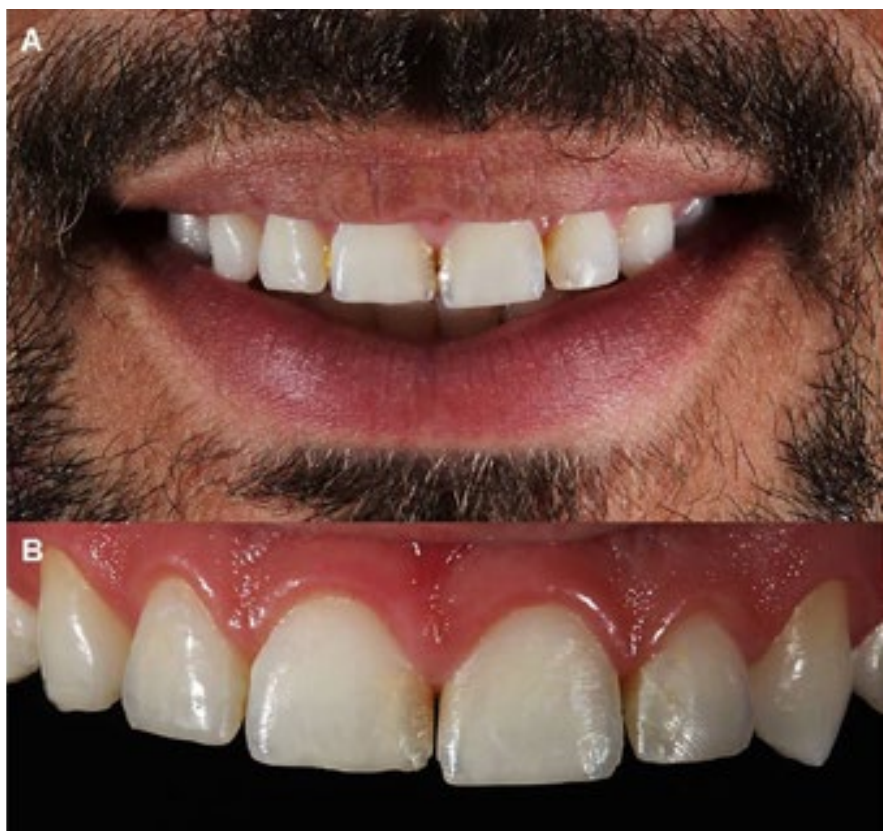

Figura 1 - Aspecto inicial; A: foto inicial mostrando a irregularidade nas incisais dos incisivos superiores; B: foto em maior aumento mostrando as restaurações insatisfatórias nas incisais e interproximais dos incisivos centrais.

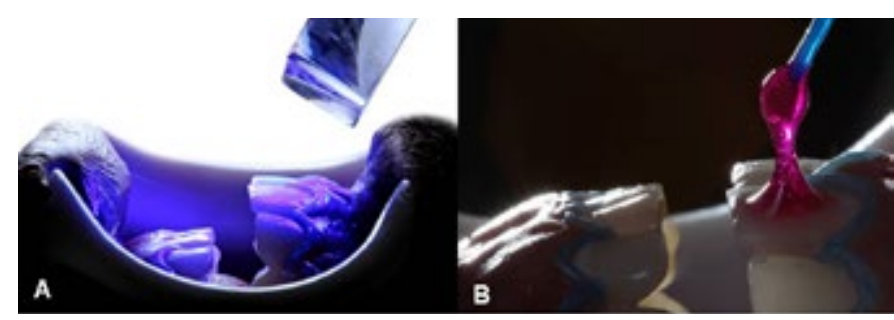

Figura 2A - Clareamento dentário; A: antes da realização do clareamento; B: resultado após as sessões de clareamento.

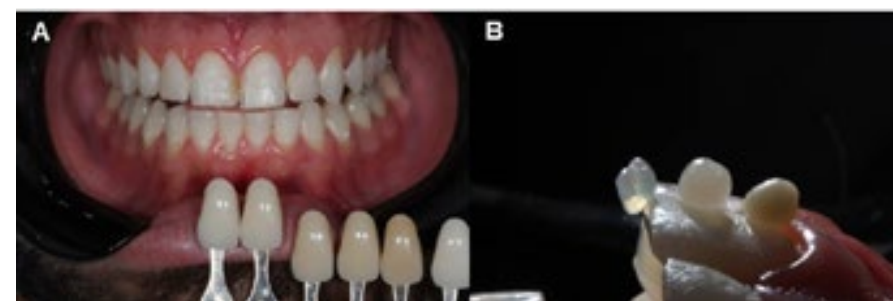

Figura 3 - Seleção de cor; A: seleção de cor com auxílio de uma escala; B: técnica de incremento de resina na vestibular sem ácido e sem adesivo.

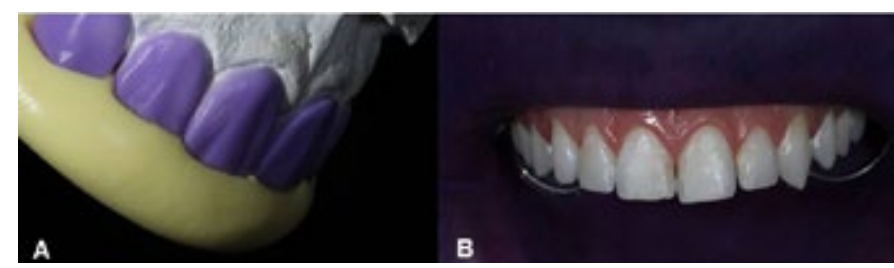

Figura 4A - Confeccção da guia palatina em silicona; B: isolamento absoluto com lençol de borracha.

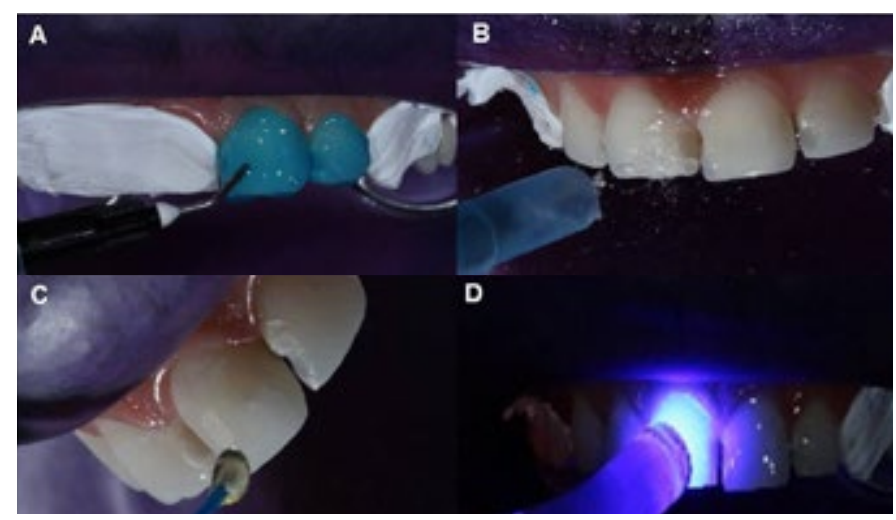

Figura 5A - Condicionamento ácido; B: lavagem por 60s; C: aplicação do sistema adesivo; D: fotopolimerização do adesivo.

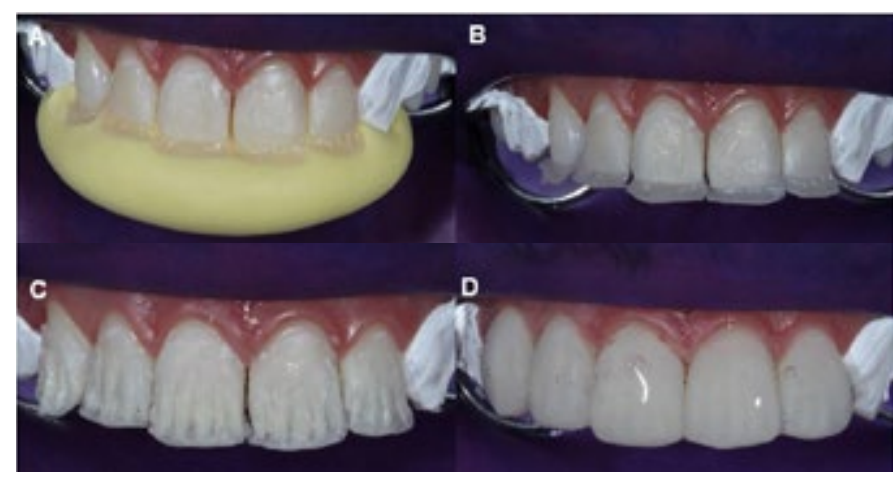

Figura 6A - Confecção das faces palatinas com a guia de silicona em posição; B: faces palatinas confeccionadas; $\mathbf{C}$ : foram colocados incrementos de resina de dentina e corpo pela técnica de estratificação de cor; D: sob a última camada foi colocado gel hidrossolúvel para bloqueio do oxigênio na fotopolimerização final. 
Na sequência, os excessos grosseiros foram retirados com pontas diamantadas para acabamento (3195F e 3118F, KG Sorensen, Cotia, Brasil) (Figura 7A) verificando os contatos oclusais em máxima intercuspidação habitual, protusiva e lateralidade direita e esquerda.

Em seguida, foram realizadas demarcações com lápis nas cores vermelho e azul, para orientar no momento de desgastar com discos Sof-lex (Pop On Kit - 3M, São José do Rio Preto, SP, Brasil), reproduzindo a anatomia secundária (Figura $7 \mathrm{~B}$ ).

Posteriormente, foi realizado polimento com borracha de silicone (Kit Dhpro, Brasil), e de feltro (Diamond-FGM, Paranaguá, $\mathrm{PR}$, Brasil) associados à pasta diamantada (Diamond-FGM, Paranaguá, $\mathrm{PR}$, Brasil), onde obteve-se adequada lisura superficial e textura das restaurações como pode ser observado na Figura 8. Após finalização do tratamento foi mostrada ao paciente a foto do antes (Figura 9A) e depois (Figura 9B) para que ele pudesse observar melhor o resultado obtido.

\section{DISCUSSÃO}

A influência de um sorriso harmônico na vida das pessoas tem contribuído para o aumento da procura por procedimentos odontológicos estéticos. A estética dental está diretamente ligada à estética facial ${ }^{7}$.

Segundo Guerra et al. ${ }^{8}$ (2017) essa maior procura pelos procedimentos odontológicos estéticos contribuiu para que a ciência odontológica desenvolvesse novos materiais, técnicas e procedimentos mais avançados que atendessem às exigências dos pacientes.

Os materiais restauradores como as resinas compostas passaram por um grande processo de evolução, aprimorando a reprodução das características naturais das estruturas dentárias ${ }^{6}$. Mendonça et al. ${ }^{9}$ (2017) afirmam que os procedimentos restauradores usando resina composta podem ser considerados um tipo de tratamento restaurador minimamente invasivo, pois, é possível preservar a estrutura dental ao máximo.

Quando comparada com a cerâmica, a resina composta apresenta vantagens como: excelente estética; longevidade aceitável; menor custo; dispensam etapas laboratoriais; técnica rápida, segura e eficaz; e ainda reversível, caso não se tenha alcançado o resultado estético desejado ${ }^{8}$.

Entretanto, de acordo com Machado et al. ${ }^{10}$ (2016) o uso da técnica direta com resina composta é limitado em situações de perdas estruturais muito extensas, pois não há remanescente dentário suficiente para oferecer a retenção adequada. Esses autores também ressaltaram que as facetas diretas em resina composta são contraindicadas em casos de apinhamento severo, oclusão do tipo topo a topo, hábitos parafuncionais e ausência de esmalte na porção cervical.

Berwanger et al. ${ }^{11}$ (2016) ainda acrescentaram que a técnica indireta utilizando cerâmica permite uma melhor estabilidade de cor e consequentemente maior durabilidade dos aspectos estéticos. No entanto, exigem um desgaste dentário mais extenso, além de ter um custo mais elevado para o paciente.

No presente caso, foram propostos ao paciente duas opções de tratamento, facetas diretas com resina compostas e facetas indiretas em cerâmica. Após passadas todas as orientações sobre os dois tipos de tratamento, o paciente optou pelas facetas diretas por ser um tratamento minimamente invasivo e de custo mais acessível.

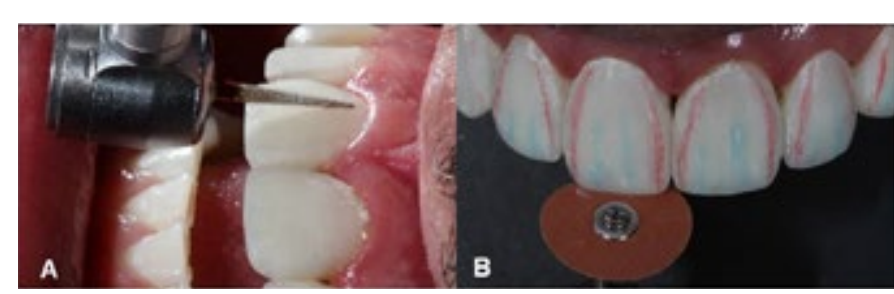

Figura 7A - Os excessos foram removidos com pontas diamantadas multilaminadas finas e extrafinas; B: demarcações com lápis de cor vermelho e azul, e desgaste com discos sof-lex reproduzindo a anatomia secundária.

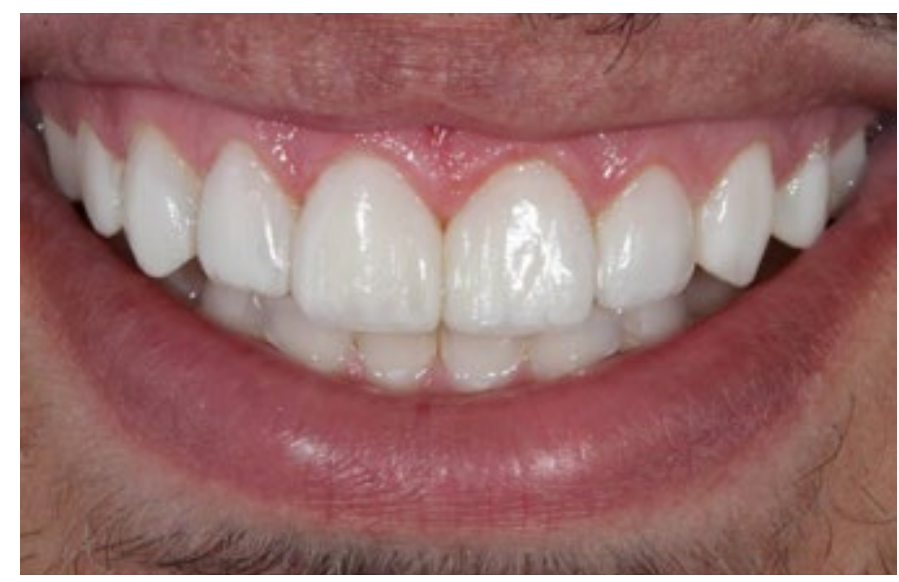

Figura 8 - Aspecto imediato do resultado final.

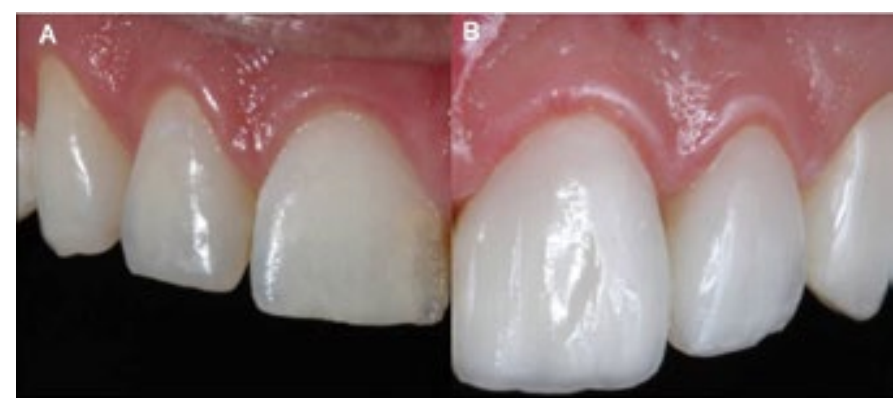

Figura 9A - Aspecto inicial; B: aspecto final.

Após a aprovação do plano de tratamento optou-se por realizar três sessões de clareamento dentário em consultório. De acordo com Veronezi et al. ${ }^{7}$ (2017) o clareamento dentário realizado em consultório permite um maior controle sob a ingestão do gel clareador e sob os danos que este pode causar aos tecidos moles. No entanto, os autores ressaltaram que o clareamento dentário caseiro apresenta vantagens como: ser autoaplicável e de menor custo para o paciente.

Finalizadas as sessões de clareamento, foram feitas as moldagens, confecção dos modelos em gessos e montagem em articulador semi ajustável para realização do enceramento de diagnóstico, o qual permite ao paciente visualizar o resultado final do tratamento. Estando de acordo com o enceramento diagnóstico oferecido, para uma reprodução mais fiel, foi confeccionada uma guia palatina de silicona que possibilitou a reconstrução da face palatina dos dentes envolvidos.

Segundo Netto e Werneck ${ }^{6}$ (2010), a guia de silicona oferece exata posição das superfícies palatina e incisal dos dentes e ainda 
reestabelece os pontos de contatos, otimizando assim a reprodução estética e o tempo clínico.

Confeccionadas as faces palatinas e incisais com auxílio da guia de silicona, as restaurações foram feitas utilizando a técnica de estratificação de cor, com intuito de alcançar maior naturalidade no resultado final. De acordo com Braga et al. ${ }^{4}$ (2016) a técnica de estratificação de cor facilita a acomodação das camadas de resina de forma natural. Além de permitir uma melhor reprodução da translucidez da estrutura dentária, criando assim um efeito óptico harmônico.

O acabamento e polimento são etapas de fundamental importância para o sucesso das restaurações em resina composta. Sendo definido como o processo de remoção das irregularidades ou excessos com a finalidade de melhorar os contornos anatômicos, promovendo regularidade à superfície da restauração. $\mathrm{O}$ polimento, por sua vez, consiste na obtenção de brilho e reflexão de luz, tornando a superfície homogênea, removendo as ranhuras geradas pelas pontas de acabamento ${ }^{12-15}$.

Menezes et al..$^{13}$ (2014) afirmaram que as pastas diamantadas apresentam eficácia tanto para o acabamento, quanto para o polimento, no entanto estas devem ser utilizadas com pontas sem abrasivos, pois, as mesmas já possuem abrasividade própria e ao serem misturadas com outros abrasivos podem interferir negativamente no resultado final.

No presente caso, para realização do acabamento foram utilizados discos abrasivos e pontas diamantadas multilaminadas de granulação fina e extrafina, buscando reproduzir as características secundárias anatômicas. E para o polimento foi utilizado disco de feltro associado à pasta diamantada, com intuito de obter o brilho final das restaurações. Finalizado o tratamento obteve-se um sorriso esteticamente harmônico e consequentemente a satisfação por parte do paciente.

\section{CONCLUSÃO}

A reabilitação de dentes anteriores com facetas em resina composta é um tratamento viável para se alcançar um resultado satisfatório.

\section{REFERÊNCIAS}

01. Sousa SJB, Magalhães D, Silva GR, Soares CJ, Soares PFB, SantosFilho PCF. Cirurgia plástica periodontal para correção de sorriso gengival associada à restaurações em resina composta: relato de caso clínico. Rev Odontol Bras Central. 2010;19(51):362-66.

02. Follak AC, Ilha BD, Ribeiro DS, Mielke JC, Buligon MP, David SDC, Durand LB. Reanatomização e Fechamento de Triângulo Negro em dentes Ântereossuperiores. Revista Dentística on line. 2012; 11(23):26-32.
03. Berwanger C, Rodrigues RB, Ev LD, Yamith A, Denadai GDA, Erhardt MCG, Souza FHDC. Fechamento de diastema com resina composta direta-relato de caso clínico. Rev Assoc Paul Cir Dent. 2016; 70(3):317-22

04. Braga AT, Monteiro DDH, Munari LS, Cornacchia TPM. Acompanhamento de restaurações em diastemas anteriores com resina composta pela técnica da barreira palatina: relato de caso. Revista Odontol Bras Central. 2016; 25(74):112-17.

05. Dantas VED, Cavalcanti YW, Carvalho WL, Pinheiro IVA, Santos AJS. Clareamento dentário como etapa prévia à restauração de dentes com alteração severa de cor. Revista Brasileira de Ciências da Saúde. 2014; 18(1):41-8.

06. Netto L, Werneck D. Resolução estética de dentes anteriores em única sessão com uso da matriz de silicone: relato de caso clínico. Revista Dentística on line. 2011; 10(22):5-8.

07. Veronezi MC, Brianezzi LFDF, Modena K, Lima MDS, Bernardi SE. Remodelação estética de dentes conoides: tratamento multidisciplinar. Revista Digital da Academia Paraense de Odontologia. 2017; 1(1):35-40.

08. Guerra MLR, Venâncio GN, Augusto CR. Fechamento de diastemas anteriores com resina composta direta: relato de caso. Faculdade de Odontologia de Lins/Unimep. 2017; 27(1):63-68.

09. Mendonça LM, Modena KS, Lima MS, Veronezi MC. Tratamento restaurador multidisciplinar para o restabelecimento da harmonia do sorriso. Rev Salusvita. 2017; 36(2):489-99.

10. Machado AC, Reinke ACMA, Guilherme FM, Zeola LF, Costa MM, Reis BR, Soares PV. Reabilitação estética e funcional com facetas diretas após histórico, de traumatismo dento-alveolar. Rev Odontol Bras Central. 2016, 25(74):154-61.

11. Campos P, Amaral D, Silva M, Barreto S, Pereira G, Prado M. Reabilitação da estética na recuperação da harmonia do sorriso: relato de caso. Revista Da Faculdade De Odontologia - UPF. 2015; 20(2):227-31.

12. Kina M, Borghi S, Fabre AF, Martins OC, Simonato LE, Boer NP, Kina J. Clareamento dental em dentes vitais: protocolo clínico em consultório. Arch Health Invest. 2015; 4(4):7-12.

13. Menezes MS, Silva FP, Borges MG, Pereira DA. Acabamento e polimento em resina composta: reprodução do natural. Rev Odontol Bras Central. 2014; 23(66):124-29.

14. Fonseca RB, Kasuya AVB, Favarão IN, Honorato IS, Santos ML, Filho-Alves LC. Técnica de estratificação e texturização superficial de resinas compostas em dentes anteriores - seis meses de acompanhamento. Clín Int J Braz Dent. 2013; 9(3):322-30.

15. Chimeli TBC, Pedreira APRV, Souza TCP, Maciel M, Paula LM, Garcia FCP. Tratamento restaurador de diastemas anteriores com restaurações diretas em resina composta: relato de caso. Revista Dentística on line. 2011; 10(20):54-57.

\section{ABSTRACT}

Objective: The present study aimed to report a clinical case of rehabilitation of anterior teeth with facets in composite resin. Case report: A male patient, 23 years-old, complained with the aesthetics of his smile. In the clinical examination, unsatisfactory restorations were observed in the incisals and interproximal of the upper central incisors. The teeth 12, 22 and 23 were in disharmony with the face. After the treatment plan, initial color registration and three dental bleaching sessions were performed. In the following visit, prophylaxis was made with extrafine pumice stone, preparation of the silicone guide by waxing, removal of the old resins, followed by acid conditioning and adhesive system. With the guide of silicone and polyester strips in place were made increments of resin composed of dentin and enamel. In the sequence, the excesses were removed with thin and extrafine 
multilaminated diamond tips, checking the occlusal contacts. Subsequently, it was polished with silicone rubber, sof-lex and felt discs, associated to the diamond paste. At the end of the treatment, an aesthetically harmonious smile was obtained and consequently patient satisfaction. Conclusion: The rehabilitation of anterior teeth with dental veneers in composite resin is a viable treatment to achieve a harmonious smile.

KEYWORDS: Esthetics dental; Dental Veneers; Smile.

\section{AUTOR PARA CORRESPONDÊNCIA}

Natália Galvão Garcia

Centro Universitário de Lavras (UNILAVRAS-MG)

Rua Padre José Poggel, 506 - Centenario, Lavras - MG, 37200-000

E-mail: natggalvao@hotmail.com 